\title{
IDENTIFICATION OF FOUR X-RAY SOURCES FROM THE INTEGRAL AND SWIFT CATALOGS
}

(c) 2018 A. A. Lutovinov ${ }^{*}{ }^{1}$, A. I. Mironov ${ }^{1,2}$, R. A. Burenin ${ }^{1}$, M. G. Revnivtsev ${ }^{1}$, S. S. Tsygankov ${ }^{3,4,1}$, M. N. Pavlinsky ${ }^{1}$, I. V. Korobtsev ${ }^{5}$ and M. V. Eselevich ${ }^{5}$

(1) Space Research Institute, Russian Academy of Sciences, Moscow, Russia (2) Moscow Institute of Physics and Technology, Dolgoprudnyi, Moscow reg., Russia (3) Finnish Centre for Astronomy with ESO (FINCA), University of Turku, Piikkiö, Finland (4) Astronomy Division, Department of Physics, University of Oulu, Finland

(5) Institute for Solar-Terrestrial Physics, Russian Academy of Sciences, Siberian Branch, Irkutsk, 664033 Russia

Received 18 March 2013

\begin{abstract}
Four hard X-ray sources from the INTEGRAL and Swift catalogs have been identified. X-ray and optical spectra have been obtained for each of the objects being studied by using data from the INTEGRAL, Swift, ROSAT, and Chandra X-ray observatories as well as observations with the RTT150 and AZT-33IK optical telescopes. Two sources (SWIFT J1553.6+2606 and SWIFT J1852.2+8424) are shown to be extragalactic in nature: the first is a quasar, while the registered X-ray flux from the second is the total emission from two Seyfert 1 galaxies at redshifts 0.1828 and 0.2249. The source IGR J22534+6243 resides in our Galaxy and is an X-ray pulsar with a period of $\sim 46.674 \mathrm{~s}$ that is a member of a high-mass X-ray binary, probably with a Be star. The nature of yet another Galactic source, SWIFT J1852.8+3002, is not completely clear and infrared spectroscopy is needed to establish it.
\end{abstract}

Keywords: X-ray sources, active galactic nuclei, X-ray binaries

\section{INTRODUCTION}

The value of astrophysical sky surveys in various 'wavelength ranges for their subsequent use in studying the physical and statistical properties 'of various populations of sources is directly related both to the completeness of the surveys themselves and to the completeness of identifying and determining the nature of the objects detected in them. At present, the most complete all-sky hard X-ray (>15 keV) surveys are the INTEGRAL '(Krivonos et al. 2010a, 2012; Bird et al. 2010) and 'Swift (Cusumano et al. 2010; Baumgartner et al. 2013) surveys. These have a very high identification completeness of the detected sources; in particular, this completeness reaches $92 \%$ in the INTEGRAL Galactic survey (Krivonos et al. 2012). Such a high percentage has been reached through the long-term work of several scientific groups in the world (see, e.g., the review by Parisi et al. 2013, and references therein; Masetti et al. 2007, 2010; Tomsick et al.

\footnotetext{
*e-mail: aal@iki.rssi.ru
}

2008, 2009), including our work (Bikmaev et al. 2006, 2008; Burenin et al. 2008, 2009; Lutovinov et al. 2012a, 2012b; Karasev et al. 2012), using soft Xray $(<10 \mathrm{keV})$, optical, and infrared observations.

This work is the next one in our program on the localization and identification of hard X-ray sources from the INTEGRAL and Swift catalogs. Four objects from the INTEGRAL Galactic survey catalog (Krivonos et al. 2012) and the Swift 70month all-sky catalog (Baumgartner et al. 2013) were included in the sample: IGR J22534+6243, SWIFT J1553.6+2606, SWIFT J1852.2+8424, and SWIFT J1852.8+3002. Apart from the results of our optical observations, we also present the results of our spectral and timing analysis for these sources obtained from INTEGRAL, Swift, ROSAT and Chandra data.

\section{OBSERVATIONS AND DATA ANALYSIS}

The objects being investigated here were detected by the IBIS/INTEGRAL (Winkler et al. 2003) and BAT/Swift (Gehrels et al. 2004) telescopes 
Table 1. List and coordinates of sources

\begin{tabular}{lcc}
\hline \hline Name & $\begin{array}{c}\text { RA } \\
(\mathrm{J} 2000)\end{array}$ & $\begin{array}{c}\text { Dec } \\
(\mathrm{J} 2000)\end{array}$ \\
\hline SWIFT J1553.6+2606 & $15^{h} 53^{m} 34.91^{s}$ & $26^{\circ} 14^{\prime} 41.8^{\prime \prime}$ \\
SWIFT J1852.2+8424A & $18^{h} 50^{m} 24.76^{s}$ & $84^{\circ} 22^{\prime} 41.0^{\prime \prime}$ \\
SWIFT J1852.2+8424B & $18^{h} 46^{m} 50.25^{s}$ & $84^{\circ} 25^{\prime} 02.2^{\prime \prime}$ \\
SWIFT J1852.8+3002 & $18^{h} 52^{m} 49.50^{s}$ & $30^{\circ} 04^{\prime} 27.3^{\prime \prime}$ \\
IGR J22534+6243 & $22^{h} 53^{m} 55.10^{s}$ & $62^{\circ} 43^{\prime} 36.8^{\prime \prime}$ \\
\hline
\end{tabular}

as weak hard X-ray $(>15 \mathrm{keV})$ sources. The typical positional accuracy of such objects for the above instruments is $4-7^{\prime}$ (Krivonos et al. 2010b; Tueller et al. 2010), which makes their identification in the optical and infrared wavelength ranges virtually impossible. The sky regions around SWIFT J1553.6+2606, SWIFT J1852.2+8424, and SWIFT J1852.8+3002 were observed by the $\mathrm{XRT} /$ Swift telescope, which allowed one to detect them in the soft X-ray $(0.6-10 \mathrm{keV})$ energy band and to improve the positional accuracy to a few arcseconds (Baumgartner et al. 2013). IGR $\mathrm{J} 22534+6243$ was first detected on the total sky map obtained during the INTEGRAL nine-year Galactic survey (Krivonos et al. 2012). The study of the archival data showed that the sky region around IGR J22534+6243 was previously observed by the ROSAT and Chandra observatories as well as by the XRT/Swift telescope and that a soft X-ray source that can be identified with the objects 1RXS J22535.2+624354, CXOU J225355.1+624336, and 2MASS J22535512+6243368 (Landi et al. 2012; Israel and Rodriguez 2012) is registered at a statistically significant level within the INTEGRAL error circle in all these observations. In addition, based on Chandra and XRT/Swift data, Halpern (2012) detected X-ray pulsations from this object with a period of $\sim 46.67 \mathrm{~s}$. In combination with the properties of the optical star (Masetti et al. 2012), this allowed IGR J22534+6243 to be presumably classified as belonging to the class of X-ray pulsars that are members of high-mass Xray binaries. The coordinates of all four objects being studied that we obtained by analyzing the XRT data are given in Table 1; the positional accuracy is $\simeq 3.5^{\prime \prime}$.

The main optical observations were carried out on the night of July 5, 2012, with the 1.5-m RussianTurkish (RTT-150) telescope using the TFOSC1 medium- and low-resolution spectrograph. For our spectroscopy, we used grism N15 with a spectral resolution of $\approx 12 \AA$ (the full width at half maximum that gives the widest

\footnotetext{
${ }^{1}$ http://hea.iki.rssi.ru/rtt150/ru/index.php?page $=$ tfosc
}

wavelength range $(3500-9000 \AA)$ and the highest quantum efficiency; the signal integration time was $900 \mathrm{~s}$ for SWIFT J1852.8+3002 and IGR J22534+6243, $1200 \mathrm{~s}$ for SWIFT J1852.2+8424A, and $1800 \mathrm{~s}$ for SWIFT J1553.6+2606 and SWIFT J1852.2+8424B. Additional optical spectroscopy for IGR J22534+6243 was performed on March 1, 2013, with the AZT-33IK telescope at the Sayansk Observatory of the Institute for Solar-Terrestrial Physics, the Siberian Branch of the Russian Academy of Sciences. For these observations, we used the UAGS spectrograph mounted at the Cassegrain focus of the telescope and equipped with a 1300 lines/mm grating, which made it possible to take a spectrum in the range $6250-6850 \AA$, near the $H \alpha$ line, with a resolution of about $4 \AA$.

We processed the optical data in a standard way, using the IRAF ${ }^{2}$ software and our own software.

For the spectral and timing analysis of the sources in the $0.2-10 \mathrm{keV}$ energy band, we used XRT/Swift and ROSAT and Chandra (for IGR J22534+6243) observational data. They were processed with the appropriate softwar 3 and the FTOOLS 6.11 software package. A hard Xray $(>20 \mathrm{keV})$ spectrum of IGR J22534+6243 was reconstructed from INTEGRAL data using software developed at the Space Research Institute of the Russian Academy of Sciences (for more details, see Krivonos et al. 2010b).

\section{RESULTS}

Most of the objects from Table 1 lie fairly high above the Galactic plane, while IGR J22534+6243, though this source is close to it $\left(b \simeq 3^{\circ}\right)$, is nevertheless far from the central Galactic regions $\left(l \simeq 110^{\circ}\right)$. Therefore, in principle, the positional accuracy $\left(\simeq 3.5^{\prime \prime}\right)$ is high enough for soft X-ray sources to be identified at optical wavelengths. On the other hand, the weak objects from the Swift catalog detected by the BAT telescope have a positional accuracy of $\sim 6-7^{\prime}$ (Tueller et al. 2010). This can lead to ambiguities even at the stage of their identification with soft X-ray sources. There are two such sources among the four objects from Table 1.

\section{SWIFT J1553.6+2606}

In the Swift 70-month catalog (Baumgartner et al. 2013), the object SDSS J155334.73+261441.4,

\footnotetext{
${ }^{2}$ http://iraf.noao.edu

${ }^{3}$ http://swift.gsfc.nasa.gov http://cxc.harvard.edu/ciao/
}

and 

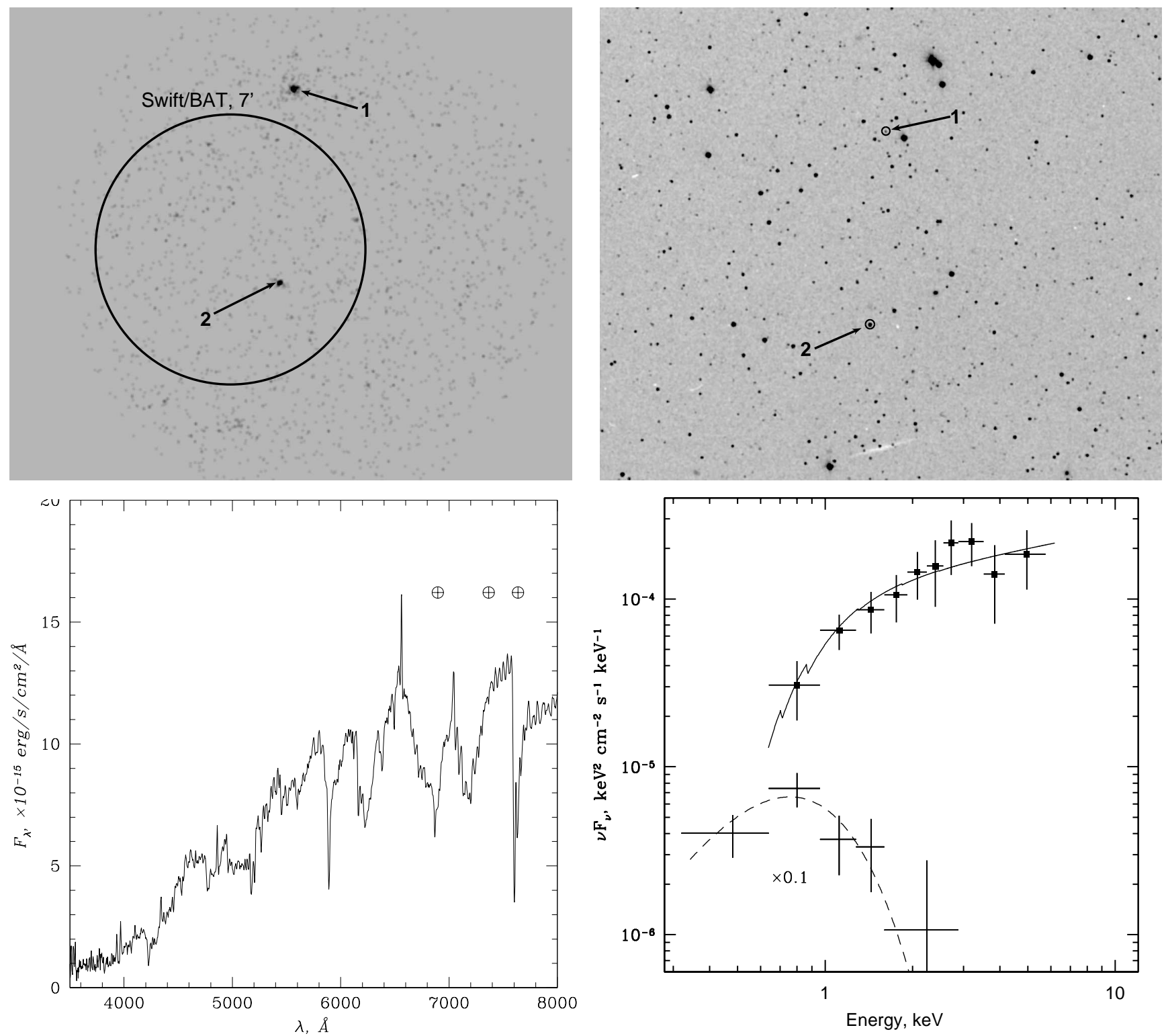

Fig. 1. (a) X-ray and (b) optical images of the sky regions around SWIFT J1553.6+2606. The circle indicates the BAT error circle of the object ( $7^{\prime}$ in radius). Numbers 1 and 2 and the arrows mark the positions of its presumed X-ray and optical counterparts. (c) The RTT-150 optical spectrum of the second source. (d) The XRT energy spectra of sources 1 (dots) and 2 (crosses). The solid and dashed lines, respectively, indicate the best fits. For clarity, the spectrum of source 2 was multiplied by 0.1 . 

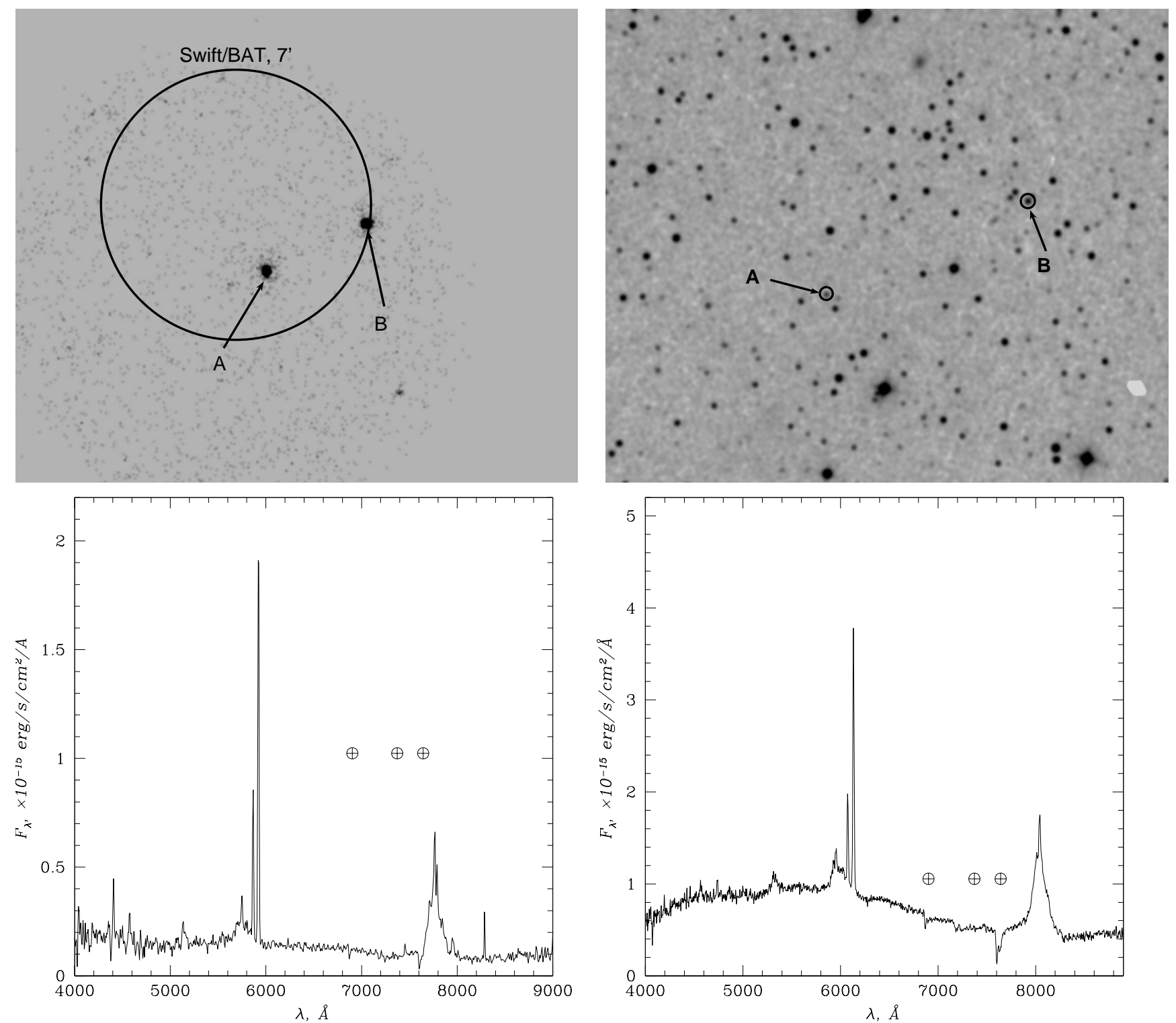

Fig. 2. (a) Soft X-ray and (b) optical images of the sky regions around SWIFT J1852.2+8424. The circle indicates the BAT error circle $\left(7^{\prime}\right)$. Letters A and B indicate the positions of its X-ray and optical counterparts. The RTT-150 optical spectra of sources A (c) and B (d). 
which is a quasar at redshift $z=0.1664 \pm 0.0013$, is specified as an optical counterpart of SWIFT J1553.6+2606. However, the separation between the BAT position of SWIFT J1553.6+2606 and the quasar's position in the sky is $\simeq 9^{\prime}$, which exceeds the BAT positional accuracy (Tueller et al. 2010). At the same time, within the Swift/BAT error circle there is another $\mathrm{X}$-ray source with coordinates $\mathrm{RA}=15^{h} 53^{m} 38.12^{s}$, Dec $=26^{\circ} 04^{\prime} 38.8^{\prime \prime}$ that can also be associated with SWIFT J1553.6+2606. This fact is illustrated in Fig. 1a, where an XRT $\mathrm{X}$-ray image of the sky region around SWIFT $\mathrm{J} 1553.6+2606$ is shown; numbers 1 and 2 indicate the quasar SDSS J155334.73+261441.4 and the second soft Xray source.

Figure $1 \mathrm{~b}$ shows the same sky region at optical wavelengths based on red Palomar Digital Sky Survey plates. Since the nature of the first object is known, we performed spectroscopy only for the second source to determine its nature. Balmer emission lines corresponding to zero redshift and broad absorption lines are clearly seen in our spectrum (Fig. 1c). Such an optical spectrum can correspond to the spectrum of an M dwarf and a star with an active chromosphere.

The XRT X-ray spectra of sources 1 and 2 (the observations were carried out several times in June-October 2010, ObsID 41177, the total exposure time is $\sim 9.3 \mathrm{ks}$ ) show a striking difference (Fig. 1d). The spectrum of the first of them is typical of quasars and can be fitted by a power-law dependence of the photon flux density on energy, $d N / d E \propto E^{-\Gamma}$, with a photon index $\Gamma=1.7 \pm 0.4$. There is absorption in the source's spectrum that exceeds the interstellar one in this direction $(\sim$ $4 \times 10^{20} \mathrm{~cm}^{-2}$; Dickey and Lockman 1990), but the significance of this measurement is not very high, $N_{H}=0.32_{-0.16}^{+0.26} \times 10^{22} \mathrm{~cm}^{-2}$. The X-ray spectrum of the second objects turns out to be considerably softer; no signal is detected above $2 \mathrm{keV}$ and the spectrum itself corresponds to blackbody radiation with a temperature of $\sim 2 \times 10^{6} \mathrm{~K}$ and a flux of $\simeq 10^{-13} \mathrm{erg} \mathrm{cm} \mathrm{cm}^{-2} \mathrm{~s}^{-1}$ in the $0.5-2 \mathrm{keV}$ energy band. Such a temperature is typical of the chromospheres of late-type stars, including $M$ dwarfs. Thus, this source (within the BAT error circle) cannot provide the hard X-ray emission from SWIFT J1553.6+2606 and the latter is a quasar at redshift 0.1664 with a luminosity of $\sim 4 \times 10^{43} \mathrm{erg}$ $\mathrm{s}^{-1}$ in the $2-10 \mathrm{keV}$ energy band.

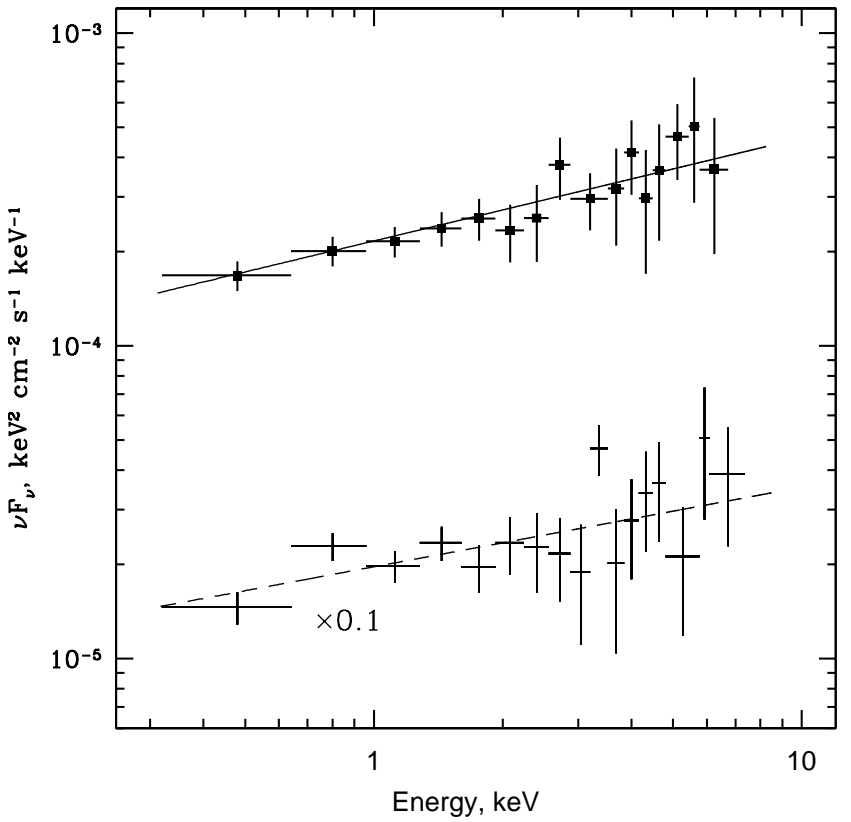

Fig. 3. XRT energy spectra of sources A (dots) and B (crosses). The solid and dashed lines, respectively, indicate the power-law best fits. As in Fig. 1, the spectrum of source $\mathrm{B}$ was multiplied by 0.1 for clarity.

\section{SWIFT J1852.2+8424}

SWIFT J1852.2+8424 is another hard X-ray source from the Swift survey with two possible soft $\mathrm{X}$-ray counterparts located within its BAT error circle (Fig. 2a). In contrast to the preceding object, the intensities of both soft X-ray sources (following Baumgartner et al. 2013, they are designated by letters $\mathrm{A}$ and $\mathrm{B}$ here) are essentially identical in the $2-10 \mathrm{keV}$ energy band $\left(F_{X, A} \simeq 7.6 \times 10^{-13}\right.$ and $F_{X, B} \simeq 9.1 \times 10^{-13} \mathrm{erg} \mathrm{cm}^{-2} \mathrm{~s}^{-1}$, respectively). In the optical wavelength range, they correspond to objects with magnitudes $m_{r, A} \simeq 17.4$ and $m_{r, B} \simeq$ 15.6 and the coordinates given in Table 3 (see also Fig. 2b). The RTT-150 optical spectra also turn out to be very similar (Figs. 2c and 2d). They exhibit sets of emission lines typical of Seyfert 1 galaxies broad Balmer hydrogen lines, narrow O[III], 4959, 5007 oxygen lines, etc. The redshifts of the galaxies measured from narrow lines are $z=0.1828$ and $z=0.2249$ for sources A and B, respectively.

The XRT X-ray spectra are typical of active galactic nuclei - they are well fitted by a simple power law with photon indices $\Gamma_{A}=1.75 \pm 0.07$ and $\Gamma_{B}=1.67 \pm 0.07$ (Fig. 3). The luminosities of the galaxies in the $2-10 \mathrm{keV}$ energy band are $L_{X, A} \simeq 0.7 \times 10^{44}$ and $L_{X, B} \simeq 1.4 \times 10^{44} \mathrm{erg}$ $\mathrm{s}^{-1}$, according to their redshifts. Thus, the X-ray 
emission from SWIFT J1852.2+8424 detected at energies $>15 \mathrm{keV}$ is the sum of the emissions from two Seyfert 1 galaxies, with the contribution from each galaxy being approximately the same.

\section{SWIFT J1852.8+3002}

According to the Palomar Digital Sky Survey plates, a fairly bright star with coordinates (J2000) $\mathrm{RA}=18^{h} 52^{m} 49.590^{s}$, Dec $=30^{\circ} 04^{\prime} 26.48^{\prime \prime}$ (Fig. 4a) and a magnitude $m_{r} \simeq 12.5$ lies almost at the center of the XRT error circle for SWIFT J1852.8+3002 (the observations in May. June 2010, ObsID 40998, the total exposure time is $\sim 9.6 \mathrm{ks}$ ). A set of absorption lines corresponding to $H \alpha, H \beta$, the CaII $\mathrm{H}$ and $\mathrm{K}$ doublet, and the Balmer jump below $\sim 3800 \AA$ (Fig. 4b) typical of F5 III stars are clearly seen in its RTT-150 optical spectrum. Comparison of the apparent and absolute magnitudes for stars of this type gives an estimate of its distance, $\sim 1 \mathrm{kpc}$.

On the other hand, the X-ray spectrum of SWIFT J1852.8+3002 fitted by a simple power law with a slope $\Gamma \simeq 1.7$ exhibits significant absorption, $N_{H} \simeq 1.6 \times 10^{22} \mathrm{~cm}^{-2}$ (Fig. 4c). This value is approximately an order of magnitude higher than the column density of the matter in our Galaxy in this direction, $N_{H} \simeq 1.4 \times 10^{21} \mathrm{~cm}^{-2}$ (Dickey and Lockman 1990), suggesting the presence of a substantial amount of matter in the binary itself possibly associated with the stellar wind from the optical companion. The X-ray flux from the source is $\simeq 10^{-13} \mathrm{erg} \mathrm{cm}^{-2} \mathrm{~s}^{-1}$ in the $2-10 \mathrm{keV}$ energy band, which corresponds to its luminosity $L_{X} \simeq$ $10^{30} \mathrm{erg} \mathrm{s}^{-1}$ for the above estimate of the distance to the binary.

Such a low luminosity in combination with an optical F5 III companion is rather unusual for Xray binaries, especially if the source's hard Xray spectrum is taken into account. A study of infrared maps and catalogs for this sky region showed that there are actually two close objects with coordinates $\mathrm{RA}=18^{h} 52^{m} 49.647^{s}$, $\mathrm{Dec}=30^{\circ} 04^{\prime} 25.44^{\prime \prime}$ and $\mathrm{RA}=18^{h} \quad 52^{m} \quad 49.431^{s}$, $\mathrm{Dec}=30^{\circ} 04^{\prime} 27.80^{\prime \prime}$ and JHK (2MASS) magnitudes $\simeq 12.25,12.08,11.82$ and $\simeq 12.71,13.75,12.11$, respectively, at the position of the bright optical star (Fig. 4d). The first of these objects probably corresponds to the optical star from the Palomar Digital Sky Survey whose spectrum was taken with RTT-150, while the second object whose position is closer to the center of the error circle for the X-ray source SWIFT J1852.8+3002 (Table 1) is its optical counterpart. However, further studies, in particular, infrared spectroscopy, are needed to ultimately answer this question and to determine the object's class. In conclusion, note that SWIFT $\mathrm{J} 1852.8+3002$ lies fairly high above the Galactic plane $\left(b \simeq 13^{\circ}\right)$, which is atypical of high mass $\mathrm{X}$-ray binaries whose vertical distribution does not exceed a hundred parsecs (see, e.g., Lutovinov et al. 2013).

\section{IGR J22534+6243}

The hard X-ray emission from IGR J22534+6243 was detected only on the total map of the Galactic plane constructed from nine-year-long INTEGRAL observations (Krivonos et al. 2012). No significant variations of the source's intensity (in particular, outbursts) were detected on its reconstructed light curve for 2003-2012 and the mean flux was $F_{X}=$ $(0.6 \pm 0.1) \times 10^{-11} \mathrm{erg} \mathrm{cm}^{-2} \mathrm{~s}^{-1}$ in the $17-60 \mathrm{keV}$ energy band.

As has already been said above, the study of the archival data showed that the sky region around IGR J22534 +6243 previously fell within the $\mathrm{XRT} /$ Swift field of view when the afterglow from GRB 060421 was investigated (ObsId. 00206257, April 21-24, 2006, the total exposure time is $72 \mathrm{ks}$ ) and the Chandra field of view in MarchApril 2009 (ObsIDs. 9919, 9920, 10810, 10811, 10812; the exposure time of each pointing was $23-28 \mathrm{ks})$. It should be noted that during the Chandra observations IGR J22534+6243 was almost at the edge of the telescope's field of view, but it was detected at a statistically significant level in all pointings. The source's coordinates measured from these observations closely coincided with those measured from XRT data (see Table 1), which allowed the optical counterpart of IGR $\mathrm{J} 22534+6243$ to be determined. It turned out to be a fairly bright object with coordinates (J2000) $\mathrm{RA}=22^{h} 53^{m} 55.130^{s}$, Dec $=62^{\circ} 43^{\prime} 36.90^{\prime \prime}$ (Fig. 5a) and a magnitude $m_{r} \simeq 13.0$, which is clearly seen in both optical and infrared (2MASS J22535512+6243368) wavelength ranges.

The RTT-150 optical spectrum of the object corresponds to a star of early spectral type O-B (Fig. 5b). At the same time, an intense Balmer $H \alpha$ emission line and a weaker $H \beta$ line are clearly seen in this spectrum. The spectrum near the emission $H \alpha$ line was taken at the AZT-33IK telescope with a spectral resolution better than that of RTT-150 (Fig. 5c). The equivalent width of the emission line is $13 \pm 1 \AA$. The $H \alpha$ emission lines with such equivalent widths are commonly observed from Be disks (see, e.g., Clark et al. 2001). Since the AZT33IK spectrum has a good resolution, we managed 

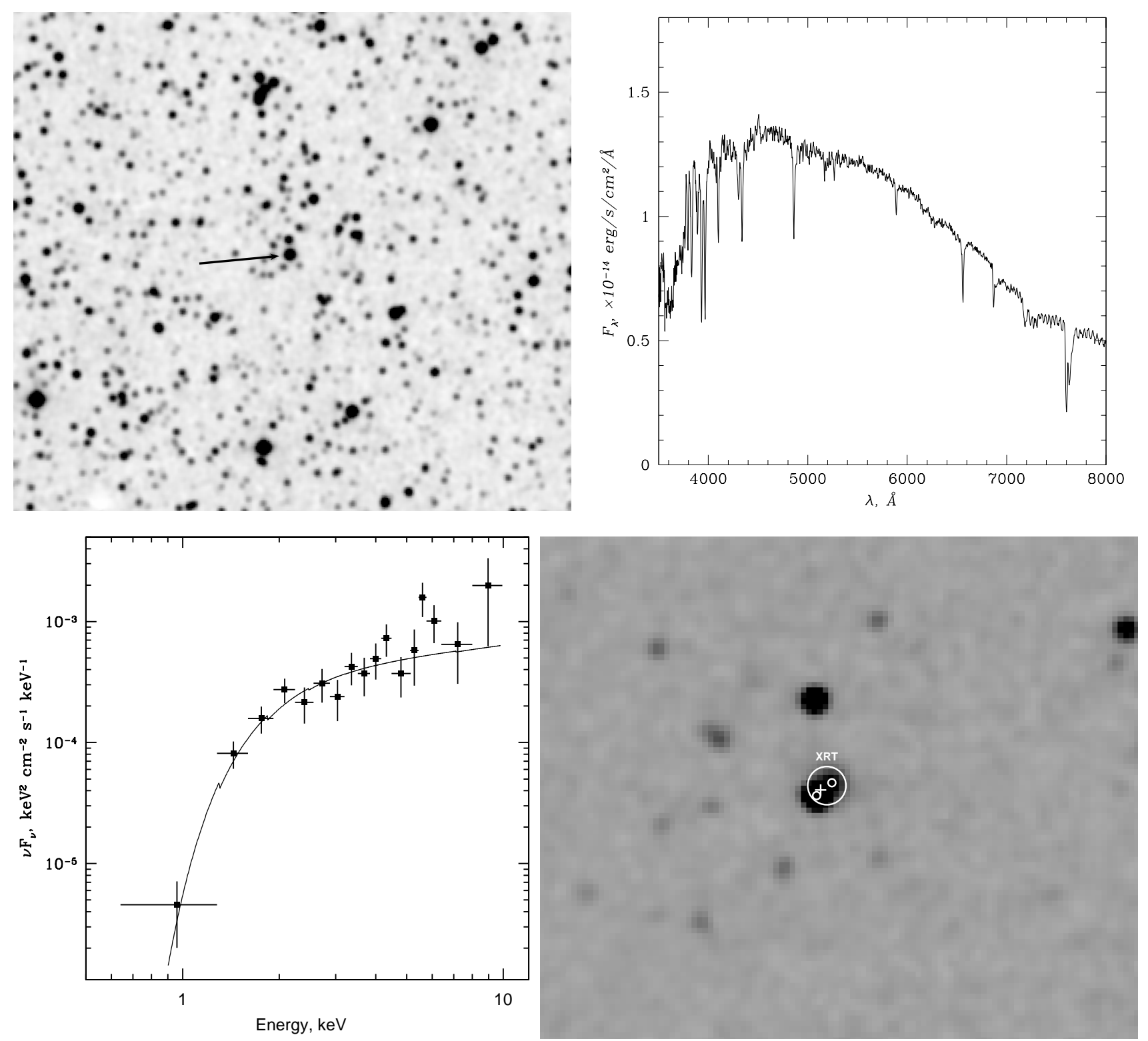

Fig. 4. (a) Optical image of the sky around SWIFT J1852.8+3002. The arrow indicates the position of the optical counterpart. (b) The RTT-150 optical spectrum of the source. (c) The XRT energy spectrum of the source. The solid line indicates the power-law best fit with absorption at low energies. (d) An enlarged infrared (2MASS, the H band) image of the sky region around SWIFT J1852.8+3002. The big white circle indicates its XRT error circle, the cross indicates the position of the optical star according to the USNO-B1 catalog, and the small circles indicate the positions of infrared objects from the 2MASS catalog. 

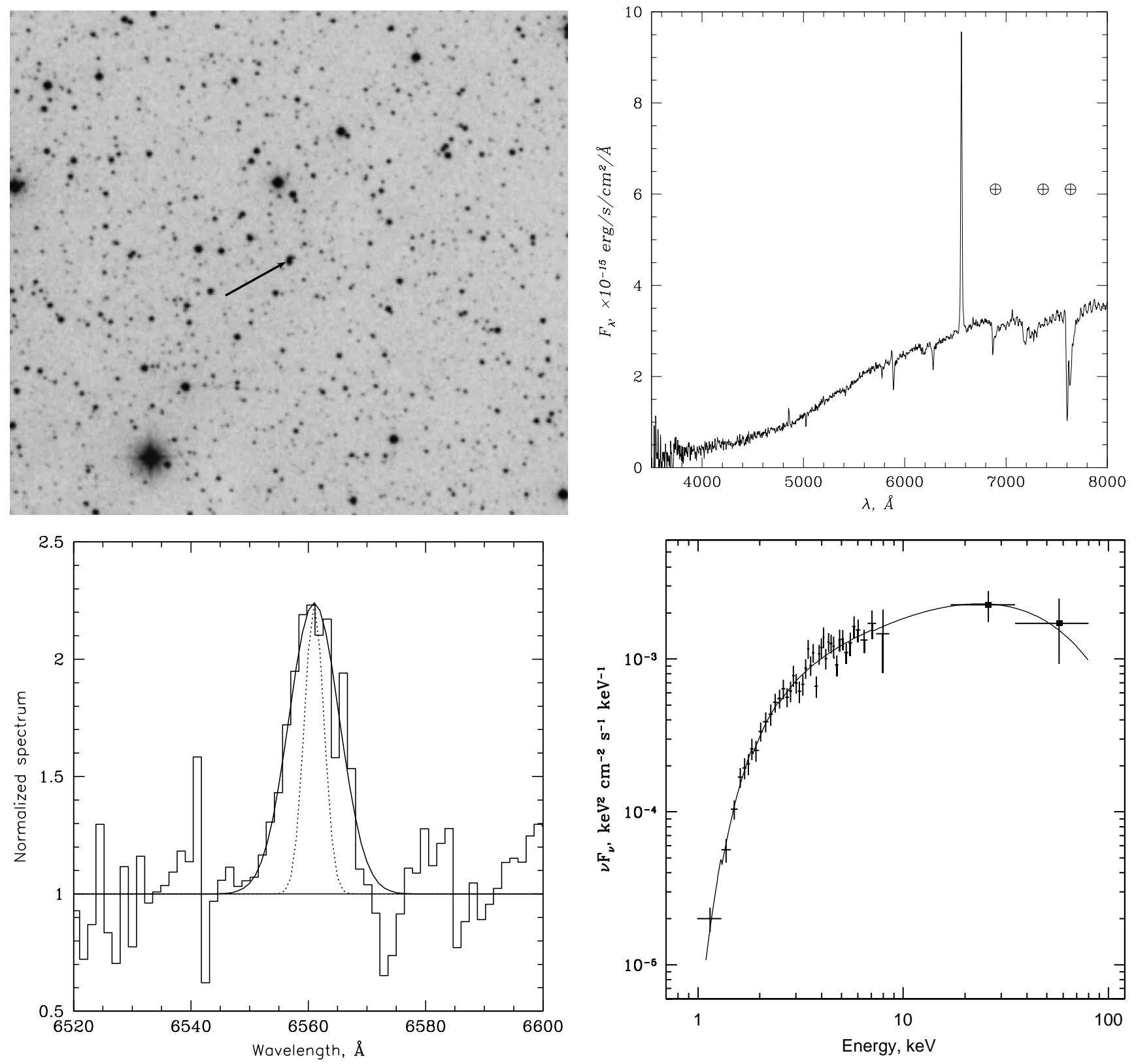

Fig. 5. (a) Optical image of the sky around IGR J22534+6243. The arrow indicates the position of the optical counterpart. (b) The RTT-150 optical spectrum of the source taken in July 2012. (c) Part of the AZT-33IK optical spectrum for the source near $H \alpha$ obtained in March 2013. The solid line indicates the best fit to the line by a Gaussian profile; the dotted line indicates the characteristic profile of atmospheric lines obtained by assuming $\Delta \lambda / \lambda=$ const. (d) The source's broadband energy spectrum from Chandra (crosses, ObsID. 10811) and INTEGRAL (dots) data. The solid line indicates the best fit by a simple power law with low-energy absorption and a high-energy cutoff. 
to detect a finite width of the $H \alpha$ emission line. At an instrumental resolution of our spectroscopic data of about $3.8 \AA$ (FWHM), the observed $H \alpha$ line width was about $4.3 \AA$. Thus, we may conclude that in the source itself the line is broadened with typical velocities of about $180 \mathrm{~km} \mathrm{~s}^{-1}$, which is also a commonly observed characteristic of the emission lines in Be systems, and is associated with a rotating equatorial disk around a Be star. It should also be noted that the line equivalent width changed by more than a factor of 2 in half a year elapsed between the RTT-150 and AZR-33IK observations (it was $\simeq 33 \AA$ in July 2012), which may be indicative of equatorial disk evolution.

Significant detection of the source by different instruments in different time intervals allowed us not only to carry out spectral and timing analysis of its emission but also to trace the evolution of its parameters. In particular, the source's intensity in the $2-10 \mathrm{keV}$ energy band remained essentially constant during the Swift and Chandra observations at $F_{X} \simeq(2.5-2.9) \times 10^{-12} \mathrm{erg} \mathrm{cm}^{-2}$ $\mathrm{s}^{-1}$, increasing only once (ObsID. 10810) to $F_{X} \simeq$ $3.1 \times 10^{-12} \mathrm{erg} \mathrm{cm}^{-2} \mathrm{~s}^{-1}$. However, given the typical flux measurement error of $\simeq(0.13-0.16) \times 10^{-12}$ erg $\mathrm{cm}^{-2} \mathrm{~s}^{-1}$, such a change may be considered insignificant. The same can also be said about the source's spectrum, which can be well fitted by a simple power law with low-energy absorption. The slope of the spectrum varies insignificantly between $\Gamma=1.35 \pm 0.14$ and $\Gamma=1.63 \pm 0.10$, becoming slightly harder, $\Gamma=1.18 \pm 0.13$, at the beginning of the series of Chandra observations (ObsID. 9920, April 16, 2009). We found no correlations between the variations of the flux from the source and the hardness of its spectrum. The parameters of the spectrum for IGR J22534+6243 determined using data from the ROSAT observatory that observed this sky region on June 18-19, 1993, (ObsID. 500321 , the total exposure time is $\sim 18.5 \mathrm{ks}$ ) agree with the Swift and Chandra measurements, but the typical measurement errors turned out to be considerably larger than those given above.

The measured absorption, $N_{H}=(2.08-2.27) \times$ $10^{22} \mathrm{~cm}^{-2}$, slightly exceeds the column density of matter in our Galaxy in this direction, $N_{H} \simeq 10^{22}$ $\mathrm{cm}^{-2}$ (Dickey and Lockman 1990). This suggest the presence of an additional amount of material in the binary itself possibly associated with the stellar wind from the optical companion. Taking into account the stability of the parameters for the X-ray spectrum of IGR J22534+6243 over a long time, we may extend it to the hard energy region
Table 2.

\begin{tabular}{ll}
\hline Data, MJD & Period, s \\
\hline 49156.08 & $46.4040 \pm 0.0008$ \\
53846.81 & $46.6148 \pm 0.0002$ \\
54937.45 & $46.6799 \pm 0.0031$ \\
54949.29 & $46.6695 \pm 0.0032$ \\
54954.07 & $46.6718 \pm 0.0024$ \\
54958.85 & $46.6723 \pm 0.0042$ \\
54959.14 & $46.6658 \pm 0.0033$ \\
\hline
\end{tabular}

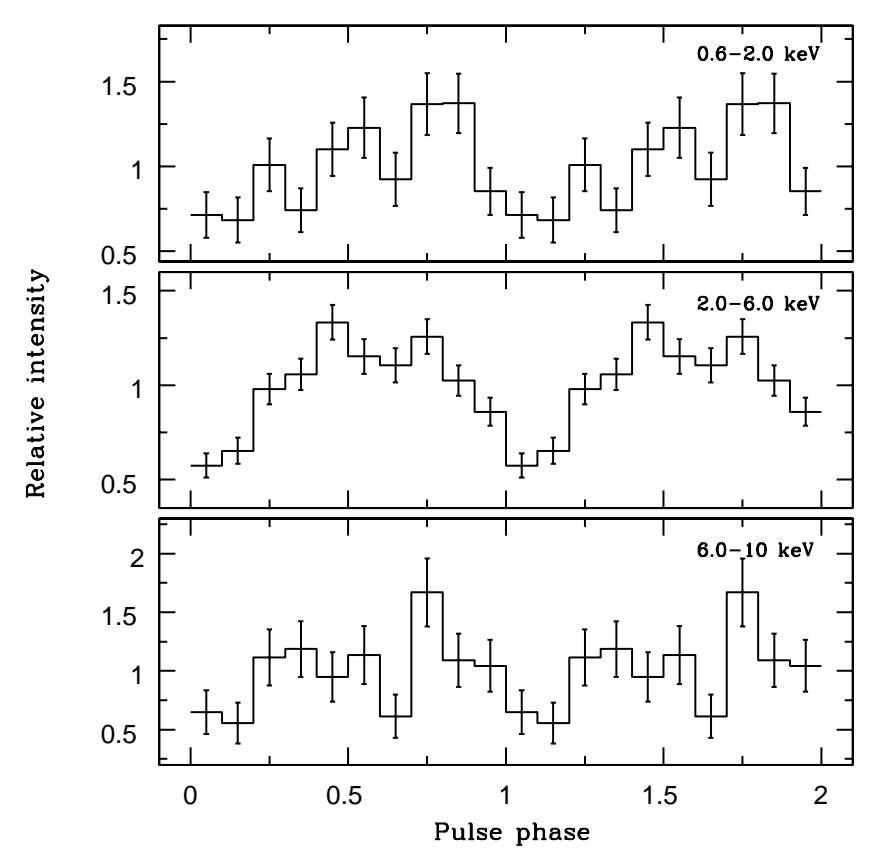

Fig. 6. Pulse profile for the X-ray pulsar IGR J22534+6243 in various energy bands obtained from one of the Chandra observations (ObsID.10811) and folded with the corresponding period.

using the INTEGRAL observational data. Such a broadband spectrum is shown in Fig.5c. Since the source is weak, a relatively significant signal from it can be registered only in broad channels. However, these measurements suggest a cutoff in the spectrum at high energies. The characteristic cutoff energy, $E_{\text {cut }} \simeq 25-30 \mathrm{keV}$, turns out to be slightly higher than that commonly observed in high-mass Xray binaries with neutron stars (see, e.g., Filippova et al. 2005). However, it should be noted that such a hard spectrum was recorded recently from low luminosity X-ray pulsars that are members of binaries with Be stars (see, e.g., Tsygankov et al. 2012; Lutovinov et al. 2012c).

Swift and Chandra data allowed Halpern (2012) to detect X-ray pulsations with a period of $\sim 46.7 \mathrm{~s}$ in the light curve for IGR J22534+6243, suggesting 
Table 3. Optical identification of the hard X-ray sources

\begin{tabular}{|c|c|c|c|c|}
\hline Name & $\overline{\mathrm{RA}}$ & $\overline{\text { Dec }}$ & Type & $z$ \\
\hline "SWIFT J1553.6+2606 & $15^{h} 53^{m} 34.734^{s}$ & $26^{\circ} 14^{\prime} 41.45^{\prime \prime}$ & QSO & 0.1664 \\
\hline SWIFT J1852.2+8424A & $18^{h} 50^{m} 25.090^{s}$ & $84^{\circ} 22^{\prime} 44.69^{\prime \prime}$ & Sy1 & 0.1828 \\
\hline SWIFT J1852.2+8424B & $18^{h} 46^{m} 49.689^{s}$ & $84^{\circ} 25^{\prime} 05.58^{\prime \prime}$ & Sy1 & 0.2489 \\
\hline SWIFT J1852.8+3002 & $18^{h} 52^{m} 49.431^{s}$ & $30^{\circ} 04^{\prime} 27.80^{\prime \prime}$ & XRB/HMXB(?) & \\
\hline IGR J22534+6243 & $22^{h} 53^{m} 55.130^{s}$ & $62^{\circ} 43^{\prime} 36.90^{\prime \prime}$ & HMXB, X-ray pulsar & \\
\hline
\end{tabular}

the presence of a neutron star as a compact object in the binary. Pulsations with a similar period were also found in the ROSAT archival data for this sky region (Israel and Rodriguez 2012). We analyzed in detail all observational data and traced the evolution of the source's pulsation period, which is presented in Table 2 (the first measurement is based on ROSAT data, the second measurement is based on XRT data, and the remaining measurements are based on Chandra data).

It can be seen from the Table 2 that in the 16-year time interval between the ROSAT and Chandra observations, the neutron star spun down significantly, with the mean spin down rate having remained almost the same over the entire period of ROSAT, Swift, and Chandra observations near $\dot{P} / P \simeq 3.5 \times 10^{-4} \mathrm{yr}^{-1}$, which is typical of $\mathrm{X}$-ray pulsars (see, e.g., Lutovinov et al. 1994; Bildsten et al. 1997). At the same time, during almost a month of Chandra observations, the pulsation period changed insignificantly, remaining mainly near $46.674 \mathrm{~s}$ within the measurement errors. The measurement errors themselves were determined by the so-called bootstrap method (for more detail, see Lutovinov et al. 2012c).

One of the most important characteristics for an X-ray pulsar is its pulse profile. As a rule, the pulse shape remains fairly stable for each specific pulsar over a long time, although it can depend on its luminosity and energy (see, e.g., Lutovinov and Tsygankov 2009). Our study of the pulse profile for IGR J22534+6243 showed that in all Chandra and Swift observations it has a similar shape (see Fig. 6) - a complex double-peak, triple-peak structure is seen in the softest $0.6-2.0 \mathrm{keV}$ energy band, which turns into one broad peak in the $2-6 \mathrm{keV}$ energy band, with the signatures of this structure remaining in it. As the energy increases, several peaks again begin to manifest themselves in the pulse profile. The latter may be due to a shortage of statistics at energies $>6 \mathrm{keV}$, while the multi-peak structure at soft energies can be a consequence of absorption in the binary. The pulsed fraction in the $2-6 \mathrm{keV}$ energy band is $\sim 40 \%$.

\section{CONCLUSIONS}

We made an optical identification of four Xray sources from the INTEGRAL and Swift catalogs and determined the nature of three of them. Two of these sources are extragalactic in nature: SWIFT $\mathrm{J} 1553.6+2606$ is a quasar at redshift $z \simeq 0.1164$; the detected flux from SWIFT J1852.2+8424 is the sum of the fluxes from two Seyfert 1 galaxies of approximately the same intensity at redshifts $z \simeq$ 0.1828 and $z \simeq 0.2489$. The other two objects are located in our Galaxy: IGR J22534+6243 belongs to the class of X-ray pulsars (with a pulsation period of $\simeq 46.674 \mathrm{~s}$ ) in high-mass X-ray binaries most likely with a Be companion; SWIFT J1852.8+3002 may also be a high-mass X-ray binary, but infrared spectroscopy is needed for the ultimate answer to the question about its nature.

Obtained results are summarized in Table 3 , which gives the coordinates of the optical counterparts of the X-ray sources, their types, and (for the extragalactic objects) redshifts.

This work was supported by the Russian Foundation for Basic Research (project nos. 1202-01265, 11-02-01328), the Presidium of Russian Academy of Sciences (programs P-21 and OFN17), the Programs of the President of Russia for Support of Leading Scientific Schools (project NSh-5603.2012.2) and the Ministry of Education and Science (Contracts N8701 and N8629). We wish to thank the TUBITAK National Observatory (TUG, Turkey), the Space Research Institute of the Russian Academy of Sciences, and the Kazan State University for support in using the 1.5m Russian-Turkish (RTT-150) telescope. We are also grateful to E.M. Churazov, who developed the IBIS/INTEGRAL data analysis methods and provided the software. 


\section{REFERENCES}

1. W. Baumgartner, J. Tueller, C. Markwardt, et al., Astrophys. J. Suppl. Ser. (2013, in press); arXiv:1212.3336.

2. I. Bikmaev, M. Revnivtsev, R. Burenin, and R. Sunyaev, Astron. Lett. 32, 588 (2006).

3. I. Bikmaev, R. Burenin, M. Revnivtsev, et al., Astron. Lett. 34, 653 (2008).

4. L. Bildsten, D. Chakrabarty, J. Chiu, et al., Astrophys. J. Suppl. Ser. 113, 367 (1997).

5. A. Bird, A. Bazzano, L. Bazzani, et al., Astrophys. J. Suppl. Ser. 186, 1 (2010).

6. R. Burenin, A. Meshcheryakov, M. Revnivtsev, et al., Astron. Lett. 34, 367 (2008).

7. R. Burenin, I. Bikmaev, M. Revnivtsev, et al., Astron. Lett. 35, 71 (2009).

8. J. S. Clark, A. E. Tarasov, A. T. Okazaki, et al., Astron. Astrophys. 380, 615 (2001).

9. G. Cusumano, V. La Parola, A. Segreto et al., Astron. Astrophys. 524, 64 (2010).

10. J. Dickey and F. Lockman, Ann. Rev. Astron. Astrophys. 28, 215 (1990).

11. E. Filippova, S. Tsygankov, A. Lutovinov, and R. Sunyaev, Astron. Lett. 31, 729 (2005).

12. N. Gehrels, G. Chinkarini, P. Giommi, et al., Astrophys. J. 611, 1005 (2004).

13. J. Halpern, Astron. Telegram 4240, 1 (2012).

14. G. Israel and G. Rodriguez, Astron. Telegram4241, 1 (2012).

15. D. I. Karasev, A. A. Lutovinov, M. G. Revnivtsev, and R. A. Krivonos, Astron. Lett. 38, 629 (2012).

16. R. Krivonos, S. Tsygankov, M. Revnivtsev, et al., Astron. Astrophys. 523, A61 (2010a).

17. R. Krivonos, M. Revnivtsev, S. Tsygankov, et al., Astron. Astrophys. 523, A107 (2010b).

18. R. Krivonos, S. Tsygankov, A. Lutovinov, et al., Astron. Astrophys. 545, A27 (2012).

19. R. Landi, L. Bassani, N. Masetti, et al., Astron. Telegram 4166, 1 (2012).

20. A. Lutovinov, S.Grebenev, R.Sunyaev and M.Pavlinsky, Astron. Lett. 20, 538 (1994)

21. A. Lutovinov and S. Tsygankov, Astron. Lett. 35, 433 (2009).

22. A. Lutovinov, R. Burenin, M. Revnivtsev, and I. Bikmaev, Astron. Lett. 38, 1 (2012a).

23. A. Lutovinov, R. Burenin, M. Revnivtsev, et al., Astron. Lett. 38, 281 (2012b).

24. A. Lutovinov, S. Tsygankov, and M. Chernyakova, Mon. Not. R. Astron. Soc. 423, 1978 (2012c).
25. A. Lutovinov, M. Revnivtsev, S. Tsygankov, and R. Krivonos, Mon. Not. R. Astron. Soc. 431, 327 (2013); $\operatorname{arXiv:1302.0728.~}$

26. N. Masetti, R. Landi, M. Pretorius, et al., Astron. Astrophys. 470, 331 (2007).

27. N. Masetti, P. Parisi, E. Palazzi, et al., Astron. Astrophys. 519, 96 (2010).

28. N. Masetti, E. Jimenes-Bailon, V. Chavushyan et al., Astron. Telegram 4248, 1 (2012).

29. P. Parisi, N. Masetti, A. Rojas, et al., in Proceedings of the 9th INTEGRAL Workshop on an INTEGRAL View of the High-Energy Sky (the first 10 years), arXiv:1302.6117.

30. J. Tomsick, S. Chaty, J. Rodriguez, et al., Astrophys. J. 685, 1143 (2008).

31. J. Tomsick, S. Chaty, J. Rodriguez, et al., Astrophys. J. 701, 811 (2009).

32. S. Tsygankov, R. Krivonos, and A. Lutovinov, Mon. Not. R. Astron. Soc. 421, 2407 (2012).

33. J. Tueller, W. Baumgartner, C. Markwardt, et al., Astrophys. J. Suppl. Ser. 186, 378 (2010).

34. C. Winkler, T. Courvoisier, G. Di Cocco, et al., Astron. Astrophys. 411, L1 (2003). 